\section{Phase measurements in MAESTRO polarimetric data from the U.K.} test sites

\section{S. QUEGAN}

Centre for Earth Observation Science,

The University of Sheffield, Western Bank, Sheffield S10 2TN, U.K.

\section{V. DUTRA}

Instituto Nacional de Pesquisas Espaciais, Avenue dos Astronautas, 1758 12201 São José dos Campos, SP, Brasil

and $\mathrm{K}$. GROVER

Centre for Earth Observation Science,

The University of Sheffield, Western Bank, Sheffield S10 2TN, U.K.

Abstract. Tests using MAESTRO data from the Reedham and Feltwell test sitcs suggest that the assumptions of a multi-variate complex zero-mean Gaussian distribution for scattering amplitudes and a linear distortion model for polarimetric data lead to a viable model for $C$-band observations, though with some deviations between theory and measurements. $P$-band observations are in gencral not consistent with this data model. When viable, the Gaussian model provides basis for defining phase information, and has implications for the statistical measures which should be used to do so. The usual mean and standard deviation statistics are shown to have undesirable characteristics when applied to phasc easurements. The effects of callbration and noise on phase measurements fron stributed targets are discussed, and other system effects on phase are notcd. nalysis of phase data from fields at the Feltwell test site shows that at $C$ ban e copolarized phase difference can discriminate between different crop types
1. Introduction

The most distinctive feature of polarimetric radars is their ability to measurc phase differences between channels. Data from the 1989 MAESTRO campaign provided an opportunity to investigate phase properties of polarimetric images from the JPL DC-8 AIRSAR system. An important aspect of the investigation concerns whether a data model based on a multi-variate Gaussian distribution for the complex scattering amplitudes, as proposed by several authors (e.g. Sarabandi 1992 Lee et al. 1991) is consistent with the data. If so, the problem of defining and measuring the phase information in the data is straightforward. We describe this model and some of its consequences. Comparisons with data suggest that $C$ banc measurements are consistent with a Gaussian model, but $\boldsymbol{P}$ band measurements are not. Properties of the theoretical distribution of the phase difference between channels, and comparison with observed histograms form the subject of Section 3 this is largely concerned with the role of the complex correlation coefficient in describing phase behaviour. The need to use a small number of statistics to summarize the phase histogram motivates a discussion of the most suitable choice of such statistics. It is shown that the mean is not a good descriptor of the representative phase for a distributed target; the mode is more appropriatc

0143-1161/94 \$10.00 (c) 1994 Taylor \& Francis Ltd 
Measures of spread should be defined relative to the mode. When the data is Gaussian, the modulus of the complex correlation coefficient provides the bes measure of spread. Section 5 consists of a short discussion of the effects of system noise on phase measurements, and observations of some pecularities in the phase images observed at $C$ band. The ability of phase to distinguish between two types of agricultural crop is considered; sugar beet and wheat show distinctly different phase responses at $C$ band, but not at $P$ band.

In this paper, the comparisons between theory and observation make use of data from the Reedham and Feltwell test sites. Both test sites are mainly agricultural, bu the Reedham test site also contains large areas of water. Imagery is available at $C$ and $L$ band for Reedham, and at $C$ and $P$ band for Feltwell. As useful ground dat were only gathered at Feltwell (a crop map and more extensive ground data were made available to us by Huntings Technical Services), most of our analysis is based on this test site. In order to have a significant number of fields on which to base our conclusions, only the dominant crop types, sugar beet and wheat are considered. Measurements were made on 22 sugar beet and 15 wheat fields at $C$ and $P$ band. $A$ limited amount of Reedham data is also used in the work presented here, because it allowed examination of the return from water surfaces and comparison of $C$ and $L$ band data.

2. The multi-variate Gaussian complex data model

For each pixel, the measurements from the JPL quadpolarized SAR at a single frequency can be represented as a 4 -vector

$$
\mathbf{O}=\left(O_{1}, O_{2}, O_{3}, O_{4}\right)^{t} \equiv\left(O_{11}, O_{21}, O_{12}, O_{22}\right)^{t}
$$

where $\mathbf{O}_{i j}$ is the observed scattering matrix element due to transmission in polarization $j$ and reception in polarization $i$, and ${ }^{~}$ denotes transpose. The extended natural targets discussed in this paper are expected to be reciprocal (which implies that the polarizability of the target is not altered by the illumination), so that for a perfect measuring system the observed data would satisfy the relation

$$
O_{21}=O_{12}
$$

In practice, this relation does not hold; the observed $H V$ and $V H$ images are different. This can be attributed to system-induced effects, for which the simplest hypothesis is a linear distortion model (van Zyl 1990)

$$
\mathbf{O}=\mathbf{M S}+\mathbf{N}
$$

where

$$
\mathrm{S}=\left(S_{1}, S_{2}, S_{3}\right)^{t} \equiv\left(S_{11}, S_{21}, S_{22}\right)^{t}
$$

is the vector of true scattering matrix elements, $\mathbf{N}$ is a noise vector and $\mathbf{M}$ is a matrix representing system effects. This paper assumes that this simple distortion model is valid.

For distributed targets, the statistical structure of the ensemble data must be described in order to discuss the potential information sources. A working hypothesis for distributed targets, based loosely on theoretical arguments (Yueh et al. 1990), is that the scattering matrix elements $S_{i j}$ obey a multi-variate complex zero-mean Gaussian distribution. This means that the probability density function (PDF) of $\mathbf{S}$ can be written as

$$
p(\mathbf{S})=\frac{1}{\pi^{3}\left|\mathbf{C}_{S}\right|} \exp \left(-\mathbf{S}^{\dagger} \mathbf{C}_{S}^{-1} \mathbf{S}\right)
$$

where the covariance matrix of the complex scattering amplitudes is given by $\mathbf{C}_{s}(i, j)=\mathbf{E}\left[S_{i} S_{i}^{*}\right], \mathbf{E}[]$ denotes expectation, ${ }^{\dagger}$ denotes conjugate transpose, and $\left|\mathbf{C}_{S}\right|$ is the determinant of $C_{S}$.

If the linear distortion model described by equation (3) is valid, then the observed data $\mathbf{O}$ will also have a zero-mean complex Gaussian distribution

$$
p(\mathbf{O})=\frac{1}{\pi^{4}|\mathbf{C}|} \exp \left(-\mathbf{O}^{+} \mathbf{C}^{-1} \mathbf{O}\right)
$$

where the covariance matrix of the observations is the $4 \times 4$ matrix

$$
\mathrm{C}=\mathrm{MC}_{\boldsymbol{S}} \mathbf{M}^{\dagger}+\mathrm{C}_{\mathrm{N}}
$$

and $C_{N}$ is the covariance matrix of the noise

There are a number of ways to test this data model, without any prior need to perform calibration. Of the five tests given below, the first depends purely on matrix properties, while the remainder are consequences of the Gaussian distribution given by equations (5) and (6).

Test 1 For large signal to noise ratio, the observed covariance matrix should have rank approximately 3 or less. This means that $\mathbf{C}$ should have at least on eigenvalue near zero. Table 1 shows two covariance matrices from the Reedham site. Only the lower triangur part of the Hermitian matrix is given; the diagent site.

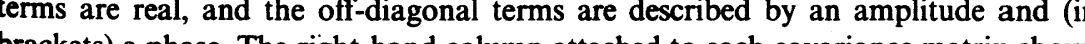
brackets) a phase. The right-hand column attached to each covariance matrix shows the relative sizes of the four eigenvalues $\lambda_{i}$, i.e., $\lambda_{i} / \Sigma_{i=1}^{4} \lambda_{i}$, expressed as a percentage That $\Sigma_{i=1} \lambda_{i}=$ Trace $(C)$, and for a Hermitian matix $\lambda_{i} \geqslant 0$.

\begin{tabular}{|c|c|c|c|c|c|}
\hline & $H H$ & $H V$ & $V H$ & $V V$ & $\lambda_{i}(\%)$ \\
\hline $\begin{array}{l}\boldsymbol{H} \boldsymbol{H} \\
\boldsymbol{H} \boldsymbol{V} \\
\boldsymbol{V} \boldsymbol{H} \\
\boldsymbol{V} \boldsymbol{V}\end{array}$ & $\begin{array}{l}5.6 \\
0.7(-110) \\
0.7(-152) \\
3.0(-18)\end{array}$ & $\begin{array}{l}0.5 \\
0.5(-42) \\
0.6(101)\end{array}$ & $\begin{array}{l}0.5 \\
0.6(140)\end{array}$ & 5.5 & $\begin{array}{r}73.9 \\
20 \cdot 8 \\
6 \cdot 2 \\
0 \cdot 1\end{array}$ \\
\hline \multicolumn{6}{|c|}{$C$ band observations and for a water body. } \\
\hline & $H H$ & $H V$ & $V H$ & $V V$ & $\lambda_{i}(\%)$ \\
\hline $\begin{array}{l}H H \\
H V \\
V H \\
V V\end{array}$ & $\begin{array}{l}0.8 \\
0.02(114) \\
0.03(50) \\
0.9(-94)\end{array}$ & $\begin{array}{l}0.14 \\
0.03(-77) \\
0.05(166)\end{array}$ & $\begin{array}{l}0.17 \\
0.05(-128)\end{array}$ & 1.6 & $\begin{array}{r}79 \cdot 5 \\
9 \cdot 5 \\
6 \cdot 6 \\
4 \cdot 4\end{array}$ \\
\hline
\end{tabular}

Table la) is for $L$ band observations of an agricultural area. The covariance matrix clearly has rank close to 3 , with one very small eigenvalue. Table $1(b)$ is for

Table 1(a). $L$ band observations from an agricultural region from the Reedham test site. The covariance matrix of the region is given in columns $2-5$ of the table; only the terms in The last column gives the relative size of the eigenvalues, as a percentage of their sum. 
$C$-band data of a water body. Here it is no longer true that the matrix has rank 3. A likely reason for this is that the noise is significant (see Section 5), but it could also indicate that the region is not truly homogeneous (if a region contains pixels from more than one distribution, the covariance matrix of each type may be of rank 3, but this may not be true of the combined dataset). However, in most of the observations the rank 3 hypothesis is tenable.

Test 2. Each channel $O_{i}$ should obey the usual speckle statistics. We therefore expect the amplitude to be Rayleigh distributed and the phase to be uniform Measurements confirm this. An implication is that for distributed targets the phase in each individual channel carries no information; it is only by examining differences between channels that phase becomes useful.

Test 3. Since the normal speckle model holds for each individual channel, the intensity data $I_{i}=\left|O_{i}\right|^{2}$ has a negative exponential distribution with mean and standard deviation equal to $\sigma_{i}^{2}$, the variance of $O_{i}$. With this notation, the complex and intensity correlation coefficients between channel $i$ and channel $j$ are

$$
\rho_{i j}=\frac{C_{i j}}{\sigma_{i} \sigma_{j}}
$$

and

$$
\rho_{i j}^{(n)}=\frac{E\left[I_{i} I_{j}\right]}{\sigma_{i}^{2} \sigma_{j}^{2}}-1
$$

The Gaussian model then predicts

$$
\rho_{i j}^{(I)}=\left|\rho_{i j}\right|^{2}
$$

(Dutra et al. 1991). Instead, measurements consistently show that the intensity correlation coefficient is somewhat greater than the modulus squared of the corresponding complex correlation coefficient. This would be expected if the regions being examined were not truly statistically uniform.

Test 4. The phase difference $\theta=\operatorname{Arg}\left(O_{i} O_{j}^{*}\right)$ between any two channels $i$ and $j$ should have PDF

$$
p(\theta)=\frac{1}{2 \pi}\left(\frac{1-\left|\rho_{i j}\right|^{2}}{1-Y^{2}}\right)\left(1+\frac{Y}{\sqrt{1-Y^{2}}}\left[\frac{\pi}{2}+\sin ^{-1} Y\right]\right)
$$

for $\theta$ in the range $-\pi<\theta \leqslant \pi$, where $\rho_{i j}$ is the complex correlation coefficient of channels $i$ and $j$ and

$$
Y=\left|\rho_{i j}\right| \cos \left[\theta-\operatorname{Arg}\left(\rho_{i j}\right)\right]
$$

(Davenport and Root 1958, Yueh et al. 1990). This means that, for any pair of channels, by measuring a single macroscopic parameter, $\rho_{i j}$, it should be possible to predict the phase difference histogram. Figures $1(a)$ and $(b)$ show comparisons between the theoretical distribution calculated from the measured correlation coefficient (solid line) and the phase difference histogram, for $C$ band data from two wheat fields at the Feltwell test site. The theoretical distributions clearly give reasonable approximations to the observations.

To put this on a more quantitative footing, goodness of fit tests were performed using the Kolmogorov-Smirnov test. The results for 22 sugar beet and 15 wheat

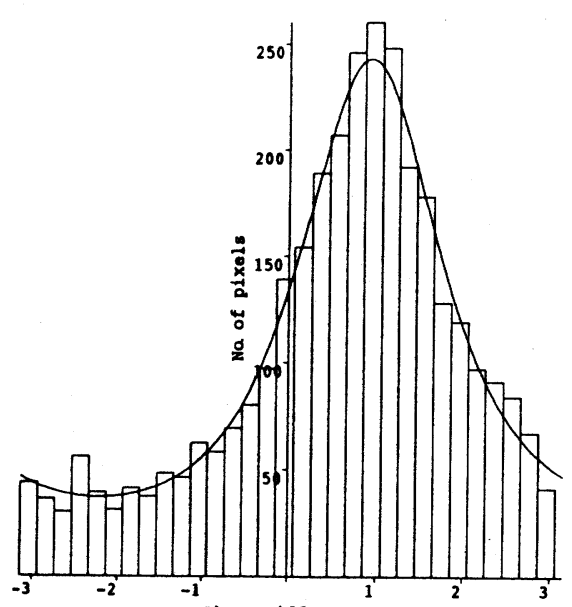

(a)

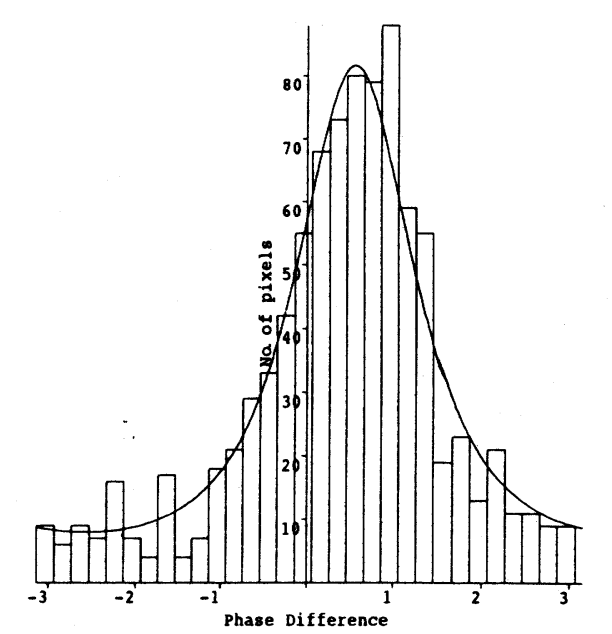

(b)
Figure 1. Comparison between theoretical phase difference probability density function (solid lines) and observed phase difference histograms. Both plots are of the $C$ band $H H-V V$ phase difference in wheat fields at the Feltwell test site. The theoretical correlation poefficient for each areap.

fields are summarized in figure 2 , which shows histograms of the degree of fit between theoretical and observed $H H-V V$ phase difference distributions for each crop type at $C$ and $P$ band. The $C$ and $P$ band results are quite different. At $C$ band, both sugar beet and wheat distributions give some faith in the viability of the theoretical distribution described by equation (11), although several fields give quite low degrees of fit. At $P$ band, very few fields are consistent with theory.

Test 5 . The amplitude ratio of any two channels $A=\left|O_{i} / O_{j}\right|$ should have PDF given by

$$
p(A)=2 \gamma_{i j}\left(1-\left|\rho_{i j}\right|^{2}\right) \frac{A\left(\gamma_{i j}+A^{2}\right)}{\left[\left(\gamma_{i j}+A^{2}\right)^{2}-4 \gamma_{i j} A^{2}\left|\rho_{i j}\right|^{2}\right]^{3 / 2}} .
$$

where $\gamma_{i j}=C_{i i} / C_{j j}=\sigma_{i}^{2} / \sigma_{j}^{2}$ (Yueh et al. 1990). By measuring the two real macroscopic parameters $\left|\rho_{i j}\right|$ and $\gamma_{i j}$ (as compared to the single complex parameter needed for the Figures $3(a)$ and $(b)$, the histogram of the amplitude ratio values can be predicted. same data as that $(b)$ show a comparison between theory and observations for the data as that used to produce figure 1.

Goodness of fit testing using the Kolmogorov-Smirnov test was again performed Tor the observed $H H / V V$ ratios, using the same fields as were used to produce the those fhown in figure 2 . The results are summarized in figure 4 , and are similar to those found for phase difference. At $C$ band, both sugar beet and wheat distribubans are reasonably consistent with the prediction of the Gaussian model. At $P$ The mew fields are consistent with theory.

The measurements shown in figures $3(a)$ and $(b)$ gave goodness of fit values of 0.65 and $0 \cdot 58$. respectively, while the corresponding values for phase difference 


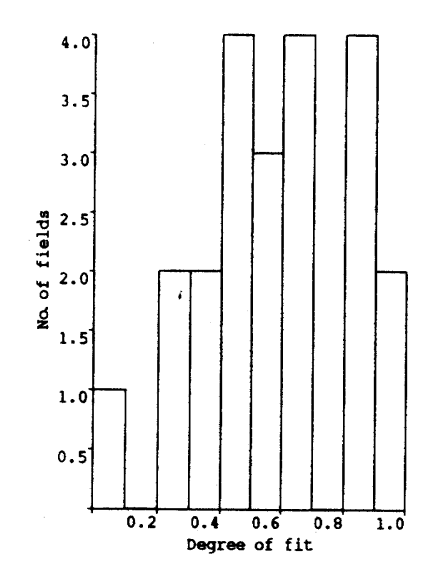

(a)

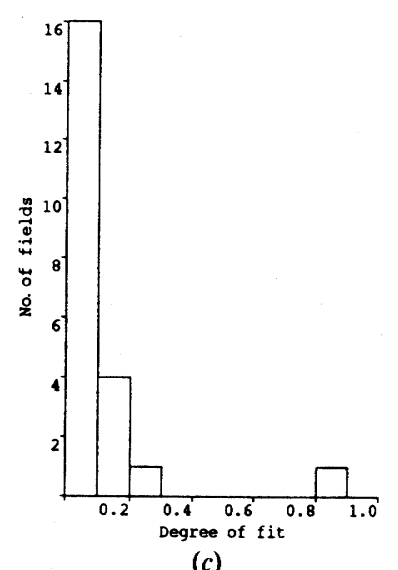

igure 2. Histograms summarizing the degrees of fit between measured and theoretica phase difference distributions, as a function of crop type and frequency. $C$ band observations from 22 sugar beet fields and 15 wheat felds are shown in figures $2(a)$ and (b) respectively; figures $2(c)$ and $(d)$ show the corresponding results for $P$ band.

measurements shown in figures $1(a)$ and $(b)$ were 0.87 and 0.34 , respectively. Comparison between the fit of the phase difference and amplitude ratio distributions for all the fields considered did not produce a consistent picture. Sometimes one of the distributions would give a good fit but not the other, sometimes both or neither would be reasonably good fits.

The results of the five tests noted above suggest that the Gaussian hypothesis is tenable for the MAESTRO data at $C$ band, but not at $P$ band. Bearing this in mind, the Gaussian model is explored further in later sections of this paper, though wherever possible the treatment is more general.

\section{The phase difference PDF}

Equation (11) shows that if the Gaussian data model is valid. then the PDF of the phase difference of any two channels is completely determined by their complex

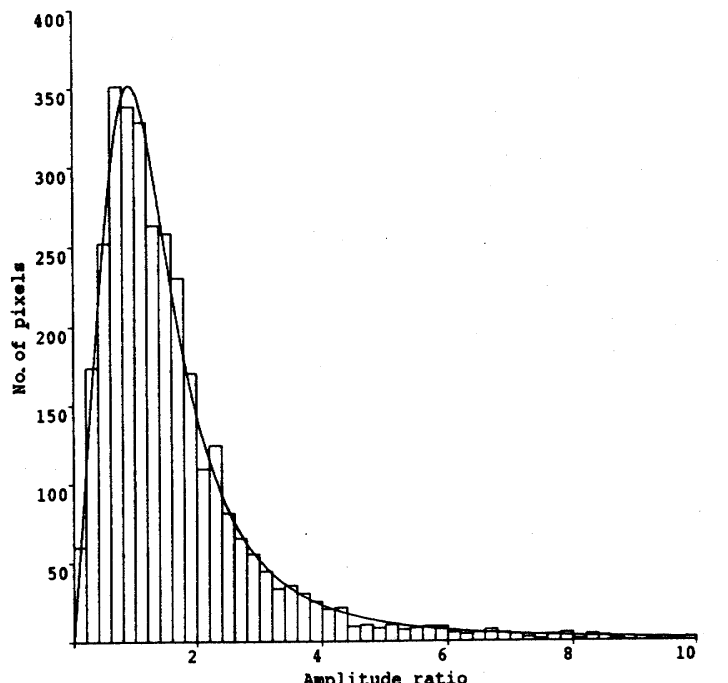

(a)

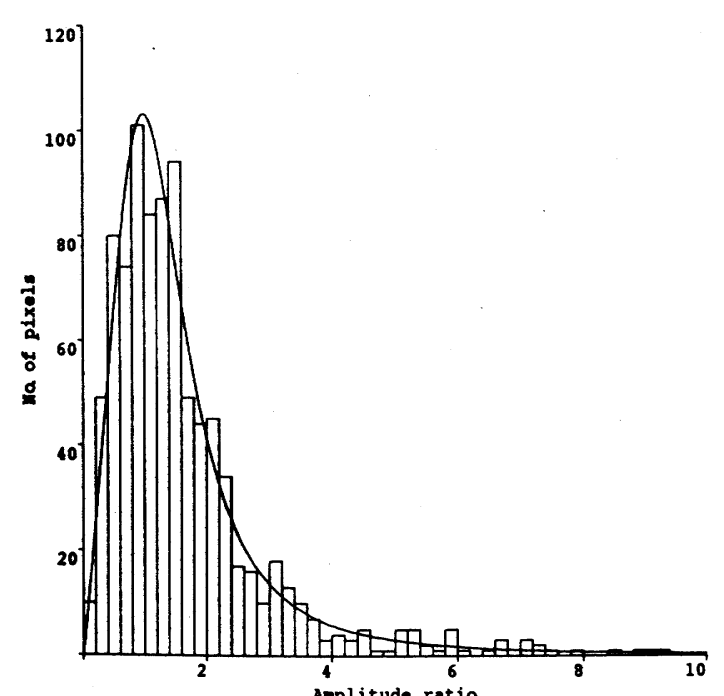

(b)

Figure 3. Comparison between theoretical amplitude ratio probability density functions (solid lines) and observed histograms, for the same-data used to produce figure 1. The theoretical curves are parametrized by the amplitude of the measured complex 


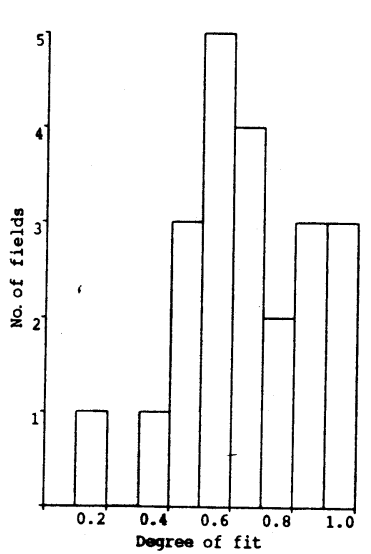

(a)

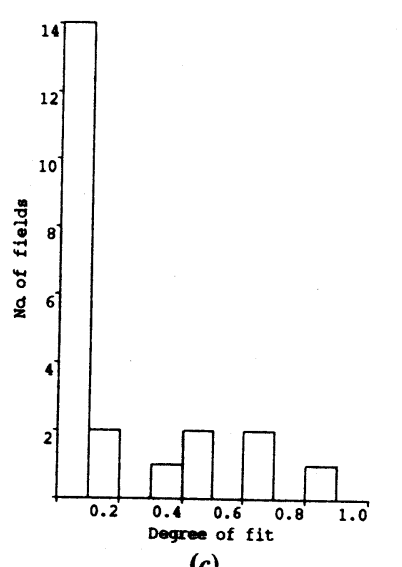

(c)
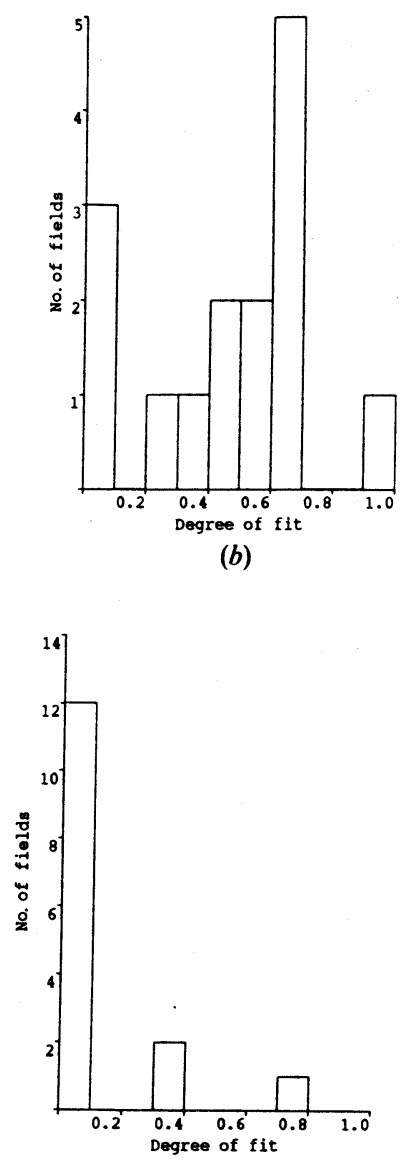

$(d)$
Figure 4. Histograms summarizing the degrees of fit between measured and theoretica amplitude ratio distributions, as a function of crop type and frequency. $C$ band $(b)$, respectively; figures $4(c)$ and $(d)$ show the corresponding results for $P$ band

correlation coefficient, $\rho$ (for convenience, the subscripts on $\rho$ have been dropped). The two real parameters $|\rho|$ and $\operatorname{Arg}(\rho)$ play different roles in this distribution. The mode is defined by $\operatorname{Arg}(\rho)$; the distribution is uni-modal and is symmetric (modulo $2 \pi)$ about its mode. The shape of the distribution is controlled by $|\rho|$, smaller values of $|\rho|$ corresponding to wider distributions. This is illustrated by figure 5 , which
(n) shows two $H V-V H$ phase difference histograms of uncalibrated $C$ band data from the Reedham area. Figure $5(a)$ is a water body, for which $|\rho|=0 \cdot 3$; figure $5(b)$ is from an agricultural area, with $|\rho|=0.85$. As figure $5(a)$ indicates, smaller values of $|\rho|$ also tend to correspond to a less well-defined mode. The extreme cases occur when (a) $|\rho|=1$ (perfect correlation or anti-correlation), in which case the phase difference

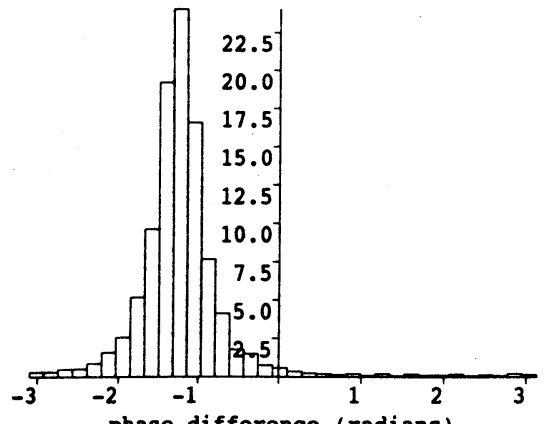

hhase difference (radians)

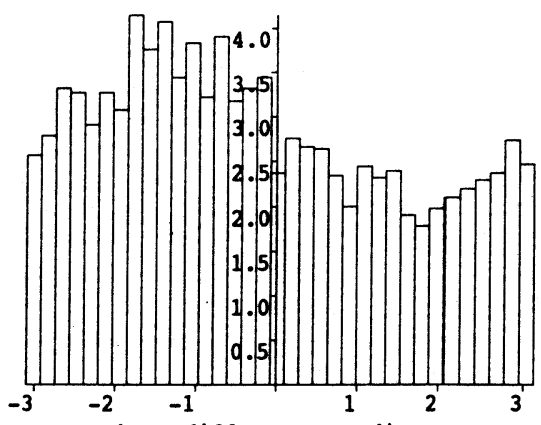

phase difference (radians)

Figure 5. Frequency histograms of the $C$ band $H V-V H$ phase difference (before calibration) for two regions from the Reedham test site. Frequency is shown as percentage. The left histogram is from an agricultural area and the right is from a
water body.

distribution is a delta function centred on $\operatorname{Arg}(\rho) ;(b) \rho=0$, when the distribution is flat, implying that no information is carried by the phase difference.

It is interesting to note (see equation 10) that $|\rho|^{2}$ can be measured from the correlation coefficient of the intensity data, so that the shape of the phase distribution can be determined purely from the intensity statistics. Hence from intensity data alone it is possible to say how well defined the phase difference will be without actually being able to measure it. The mode of the distribution is the extra parameter introduced by having polarimetric data available.

The discussion above has indicated that not all regions give phase difference histograms with good fits to the theoretical PDF, even at $C$ band. Tests were performed to assess whether, in such circumstances, the correlation coefficient still gives a good description of the phase behaviour, and whether its phase coincides with $\operatorname{Arg}(\rho)$. In order to test large numbers of distributions, automatic methods were used. This was complicated by the cyclic nature of phase, as discussed further in section 4. The procedure followed was first to find the mode of the PDF using the method described in Press et al. (1986). The distribution was then cyclically shifted (modulo $2 \pi$ ) to place the mode at the origin. If the distribution is symmetric (modulo $2 \pi$ ), the shifted distribution will have mean 0 ; it will also be near zero if it is sharply beaked. The 'peakiness' of the distribution was tested by the kurtosis measure (Press cal. 1986). Kurtosis is of non-dimension was tested by the kurtosis measure (Press distribution, relative to a Gausian distribution (which has a the sharpness listribution is sharply peaked, the kurtosis is a large positive value. In this of 0 ). If a a a well-defined phase. If the distribution is alat, kurtosis will be negtive, and there in not well defined. H. The

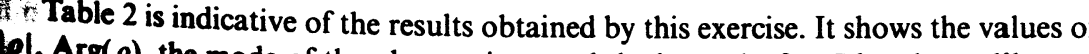
data from the mode of the observations and the kurtosis, for $C$-band uncalibrated calculating the Reedham area. Also shown is the 'shifted mean', $m^{\prime}$; this is found by the mode. As mean of the shifted distribution, then shifting it back by the value of distributio. As discussed above, to some extent it reflects the symmetry of the for reasion, though imperfectly. The mean of the original distribution is not shown. or reasons discussed in Section 4. Examples are given of three cases, riz. high. 
Table 2. Examples of the relations between the amplitude and phase of the complex correlation coefficient $(\rho)$, the mode, the shifted mean $\left(m^{\prime}\right)$ (see text), and kurtosis, for an area of uncalibrated
$m^{\prime}$ are given in degrees.

\begin{tabular}{lccccc}
\hline & $|\rho|$ & $\operatorname{Arg}(\rho)$ & Mode & $m^{\prime}$ & Kurtosis \\
\hline$H V-V H$ & 0.95 & $-73 \cdot 1$ & -75.9 & -72.8 & 8.93 \\
$H H-V V$ & 0.51 & -91.3 & -96.4 & -93.8 & -0.18 \\
$H H-H V$ & 0.03 & -16.1 & -139.7 & -137.8 & -1.22 \\
\hline
\end{tabular}

medium and low values of $|\rho|$. Large $|\rho|$ is illustrated by the phase difference of the $H V$ and $V H$ channels. This gives very high kurtosis, with the values of the mode, $m^{\prime}$ and $\operatorname{Arg}(\rho)$ all being close. These values are still close together for the $H H$ and $V V$ channels, which exhibit a medium value of $|\rho|$; the kurtosis suggests a near-Gaussian shape. The $H H$ and $H V$ channels yield a low correlation, and the kurtosis value suggests a flat distribution (kurtosis has a value of -1.2 for a uniform distibution). For such cases, the phase mode is not well defind and paral

These results These results are typical of all the $C$ band data examined. $\operatorname{Arg}(\rho)$ is a good indicator of the phase difference mode unless the value of $|\rho|$ is low. This conclusion is not dependent on there being a good fit between the observed and theoretical distribution. The observations show that when larger numbers of pixels are available, the correspondence between the observed mode of the phase difference distribution and $\operatorname{Arg}(\rho)$ improve, even when $|\rho|$ takes values as low as $0 \cdot 2$. This is not too surprising; it is the nature of sampling statistics. However, it means that at the moment it is not possible to specify how low $\rho$ can be while remaining a reliable indicator of the mode. Further analysis of this question using the MAESTRO dataset is complicated by the range dependence observed in the $C$ band phase correlation length (see Section 5). This effect implies that the number of independent phase measurements available to estimate $|\rho|$ varies with range. At $P$ band, the mode of the observed phase difference distribution and $\operatorname{Arg}(\rho)$ were still in many case close together, but in 7 out of the 37 fields examined at the Feltwell site, the mode of the $H H-V V$ phase difference was more than $10^{\circ}$ different from $\operatorname{Arg}(\rho)$.

\section{The choice of phase statistic}

The phase difference histogram is a complete description of the information contained in phase (up to spatial correlation), but does not provide a convenient summary of that information, and it is desirable to use macroscopic statistics. For a model-based approach, using equation (11), all the phase information is described by $\operatorname{Arg}(\rho)$ (since $|\rho|$ can be derived from pure amplitude data, it contains no extra information on phase). As has been shown, this ideal approach can break down if the observations do not fit the model, or if $|\rho|$ becomes small. If more general statistical descriptors are to be used, it is necessary to choose the most appropriate ones.

The usual measures for representative value and spread of data are the mean and the standard deviation. These have serious drawbacks when applied to phase data, leading to counter-intuitive results. Before carrying out a more quantitative treatment, a simple example illustrates these problems. Consider the two phase distribu-

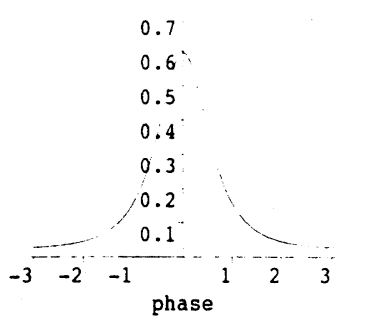

(a)

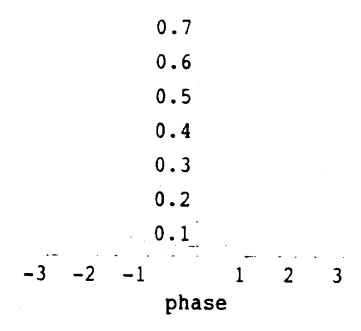

(b)

Figure 6. Two phase distributions corresponding to the same distribution of phase aroun the unit circle, but in case $(b)$ rotated by $\pi$ radians compared to case $(a)$.

tions illustrated in figure 6. Phase measurements can be represented as points on the unit circle. The distributions shown give identical distributions around the unit circle, except that in one case the points cluster round 0 , in the other round $\pi$. This is not at all well reflected in the mean and standard deviation. In fact both distributions have a mean of 0 ; in case (a) there is a low standard deviation, while in distibution, while in in this conveying the concepts they are meant to describe. in this conveying the concepts they are meant to describe.

More quantitative results are possible by making the following assumptions about the phase PDF:

(a) The PDF $p(\theta)$ has a single maximum (the mode) $\phi_{0}$;

(b) The PDF can be described as a shift (modulo $2 \pi$ ) of a PDF $p_{0}(x)$ which is symmetrical about 0 ;

(c) The function $p_{0}(\theta)$ monotonically decreases in the range $0 \leqslant \theta \leqslant \pi$.

These assumptions are consistent with the theoretical PDF of equation (11), but are more general.

It can then be shown that $\theta$ has mean

Td variance

$$
\langle\theta\rangle= \begin{cases}\phi_{0}-\int_{\pi-\phi_{0}}^{\pi} p_{0}(x) d x & \text { if } \phi_{0} \geqslant 0 \\ \phi_{0}+\int_{\pi-\left|\phi_{0}\right|}^{\pi} p_{0}(x) d x & \text { if } \phi_{0}<0\end{cases}
$$

Were $\sigma_{0}^{2}$ is the variance of $p_{0}(\theta)$, and

$$
\sigma_{\theta}^{2}=\sigma_{0}^{2}+4 \pi^{2} I_{0}\left(1-I_{0}\right)-4 \pi I_{1}
$$

$$
I_{k}=\int_{x-\mid 100}^{x} x^{k} p_{0}(x) d x
$$

$6=0,1$. These expressions for the mean and standard deviation of phase can b now illustrated both statistics have several properties which are undesirable, as is now illustrated.

If the distribution is rotated around the unit circle, the mean (unlike the mode) fixed and the with the distribution, but more slowly. In fact. if the shape of $p_{0}(\theta)$ is fixed and the mode increases from 0 , it can be seen that 
$\frac{d\langle\theta\rangle}{d \phi_{0}}=1-2 \pi p_{0}\left(\pi-\phi_{0}\right)$

which means that $\langle\theta\rangle$ maximizes when $p_{0}\left(\pi-\phi_{0}\right)=1 / 2 \pi$. After this point, though the distribution does not change its shape and the mode continues to increase, the mean decreases. This, in itself, is counter-intuitive, but there are other undesirable consequences.

(a) The mean is less sensitive than the mode as a discriminator of distributions. Two distributions of exactly the same shape but with different modes can even have the same mean.

(b) Except for delta-function distributions, the mean cannot occupy the whole of the range $(-\pi, \pi]$. As distributions become wider, the range of possible values becomes increasingly restricted (the limiting case is the uniform distribution, which is invariant under rotation and for which the mean is confined to a single point, 0 ).

It is also possible to show that if the mode increases from 0 while the shape of the distribution remains unchanged, then $\sigma_{\theta}^{2}$ also increases, reaching a maximum value of

These defects in the standard statistical measures become serious only when the mode departs significantly from 0 , and/or the phase distributions are not very narrow. For the surface types examined in this paper, these problems are not major. However, for wooded areas at $P$ band, where large values of the phase difference can occur, the above analysis suggests that the mean and standard deviation of phase are not the right measures to use. Perhaps the most telling point is that the mean and standard deviation of phase do not reflect the physics; this is carried by the correlation coefficient (see, for example, Kuga et al. 1990), which is not simply related to these quantities.

The most informative measure of representative value is the mode, unless the distribution is close to uniform, when the mode becomes ill-defined. In this case, there is effectively no phase information, and the assignment of zero phase to the distribution is arbitrary. on the mode; in the Gaussian case, the natural measure to use is $|\rho|$.

\section{Noise and system effects on phase measurements}

If noise is present in the data, then the observed complex correlation coefficient has modulus

$$
\left|\rho_{i j}\right|=\frac{\left|C_{i j}\right|}{\sqrt{\left(C_{i i}+N_{i i}\right)\left(C_{j j}+N_{j j}\right)}}
$$

where it is assumed that the noise in different channels is uncorrelated and has average power $N_{i i}$ in channel $i$. It is clear that noise will cause the correlation coefficient to decrease. This leads to a loss of dynamic range in the amplitude data, and widens the phase distribution, so that the phase measurements become less reliable. Such effects would only be expected when the noise becomes significant, and hence are more likely in the cross-polarized channels. Evidence for such effects in the data is illustrated by the lower histogram in figure 5. The wide $H V-V H$ phase difference histogram shown here is from a water body, for which the returned powe in the cross-polarized channels is very low. In fact, the $H V-V H$ phase difference histograms are always much wider in the $C$ band MAESTRO data when the return becomes low, as compared with brighter regions. Exactly similar behaviour wa noted in the $C$ band data from the 1989 Goldstone campaign and, to a much lesse extent, in $L$ band MAESTRO data. The fact that this is true for all targets with low cross-polarized power suggests that it is a noise effect, and that in this case noise cannot be neglected. Cross-talk can also widen the histogram, but its effects depend only on ratios of the terms in the scattering matrix, in the absence of noise (Quegan 1992). Hence cross-talk should not give rise to the observed brightness dependence of the phase histograms.

$C$ band phase data also suffer from another system defect which can be clearly seen in figure 7 , which is an image of the $\mathrm{HH}$ phase from a $1024 \times 750$ section of the Feltwell data. There are obvious bands in the phase, running in the azimuth direction Such banding is observed in all channels in the $C$ band int direction. Such banding is observed in all chanels in the $C$ band data. The banding does mean phase calculated along each range line, as a function of range. As expected, the mean phase is approximately zero everywhere. The perceived banding instead corresponds to changes in the local correlation of the phase. The source of this effect is unknow, it could be an indication of a mismatch in the range compression, but this appears unlikely. Though spatial correlation of phase is a possible information

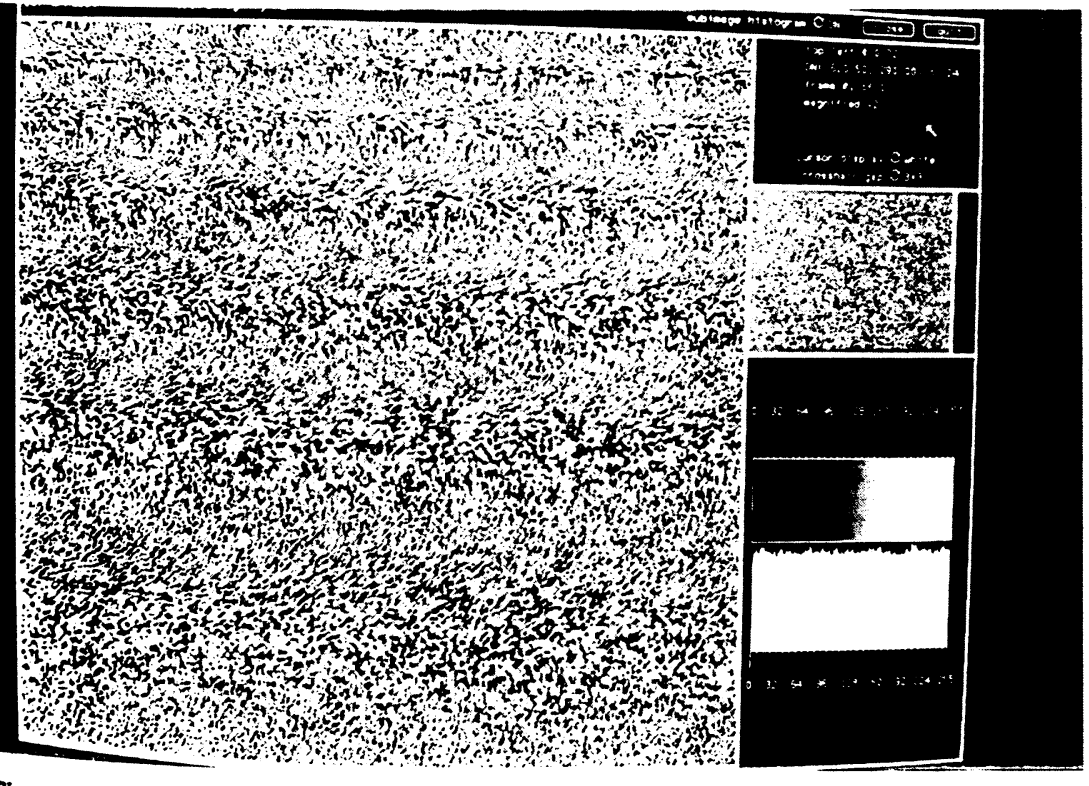

Figure 7. An image of the phase of $C$-band $H H$ observations for a $1024 \times 750$ (azimuth $\times$ range) section of the Feltwell data. Near range is at the top of the figure. The 


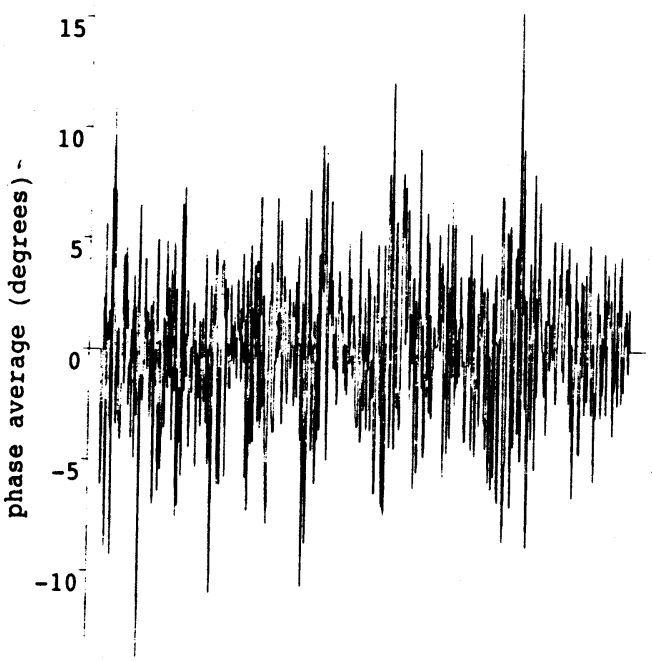

$-15$

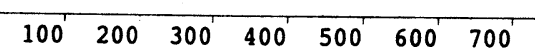

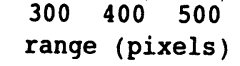
Figure 8. A plot of the mean phase along lines of constant range, as a function of range,
corresponding to the data shown in figure 7 .

source in polarimetric data, in this case it appears to be related to system performance, rather than the real world. In this case, the changing phase correlation length implies that the accuracy of phase measurements is range dependent.

\section{Phase difference as a discriminator}

The properties of the data discussed up to now have not been dependent on data calibration, only on the underlying scattering matrix data being Gaussian and the linear distortion model (equation 3) being valid. However, before discussing phase difference as a discriminator, the calibration steps necessary to produce reliable absolute phase difference measurements on this dataset need to be considered

For recovery of absolute phase difference information, cro calibration are needed Cross-talk calibration (which includes a phase and phase can be carried out using only distributed (argts, under the assumption thet scene is dominted by areas dhe scene is dominated by areas displaying azimuthal symmetry. This condition implies
that $\mathbf{C}_{\boldsymbol{s}}$ has the form

$$
\mathbf{C}_{S}=\left[\begin{array}{ccc}
\sigma_{11}^{2} & 0 & \sigma_{11} \sigma_{22} \rho_{\mathrm{co}} \\
0 & \sigma_{21}^{2} & 0 \\
\sigma_{11} \sigma_{22} \rho_{\mathrm{co}}^{*} & 0 & \sigma_{22}^{2}
\end{array}\right]
$$
where the power in each channel is given by $\sigma_{i j}^{2}=E\left[\left|S_{i j}\right|^{2}\right]$ and $\rho_{\mathrm{co}}=E\left[S_{1} S_{3}^{*}\right] / \sigma_{11} \sigma_{22}$
is the copolarized complex correlation coefficient (Nghiem et al. 1992). This assumption provides a basis for the cross-talk calibration methods described by van yl (1990), Klein and Freeman (1991) and Quegan (1992).

Cross-talk calibration drives the data into a form where azimuthally symmetric distributed targets have covariance matrices of the form (20). Since the correlation coefficients of the copolarized and cross-polarized channels are zero, the phase difference histograms of these channels then become flat, implying that they supply no information. The only phase information comes from the copolarized channels. It has already been shown in Section 3 that the width of the $H H-V V$ phase difference PDF is dependent on $\left|\rho_{\text {co }}\right|$ (at least at $C$ band). An important point noted in Quegan (1992) is that $\left|\rho_{\text {co }}\right|$ is unaffected by the linear distortions induced by the system. It can be correctly inferred from the observed covariance matrix, without calibration, and is given by

$$
\left|\rho_{\mathrm{co}}\right|=\left|\frac{C_{14}}{\sqrt{C_{11} C_{44}}}\right|
$$

Hence it is possible to establish how well defined the copolarized phase difference is by using uncalibrated data.

By contrast, measurements of absolute phase difference require correction for the phase change induced by cross-talk, followed by a further phase correction step. Correction for cross-talk is discussed in Quegan (1992); it involves substracting $103^{\circ}$ from the observed phase $H H-V V$ phase difference at $C$ band, and adding $70^{\circ}$ at $P$ band. (The corresponding value at $C$ band for Reedham is approximately $75^{\circ}$; there are no $P$ band data available for this site). Using the methods described by Zebker and Lou (1990) to define the phase calibration step requires the assumption that the $H H-V V$ phase differences are known for some regions in the imagery. It is not clear that the data made. As a result the a relevant. phase calibration, since all the falds ex phined diffe at roughy the same range, the phase albrato would shift all same amount. This means that variations in the phase values between different crop For theal differences in backscattering behaviour.

For the 22 sugar beet and 15 wheat fields on which measurements were made, the $H H-V V$ phase difference distributions of the $C$ and $P$ band data were examined for evidence that phase could be used as a discriminator. All the $C$ band phase difference distributions were unimodal and the measured values of $\operatorname{Arg}\left(\rho_{\text {co }}\right)$ agreed closely with the observed mode of the distribution. For sugar beet, $\left|\rho_{\text {col }}\right|$ occupied a narrow range (Irom 0.57 to 0.70 ), while for wheat $\left|\rho_{\text {co }}\right|$ was generally lower and more widely apread, with a range from 0.23 to 0.63 . At $P$ band, the picture was not as clear. gain the distributions were unimodal, but, as noted in Section 3, there were severa cases of an offset between $\operatorname{Arg}\left(\rho_{\mathrm{co}}\right)$ and the observed mode of the histogram. The range of values of $\left|\rho_{\text {cee }}\right|$ for sugar beet was 0.22 to 0.79 , while for wheat it covered the whole range from 0.00 to 0.94 .

Figure 9 shows histograms of the measured values of $\operatorname{Arg}\left(\rho_{c o}\right)$. Figures $9(a)$ and (c) are for $C$ band and show $A r g$ for sugar beet and wheat $\left(\rho_{c o}\right)$. Figures $q(b)$ and $(d)$ are the corresponding results for $P$ band. At $C$ band sugar beet values are closely grouped around $60^{\circ}$. Wheat exhibits a wider spread of values though the majority of values around $60^{\circ}$. Wheat exhibits a wider spread of vage, though the 


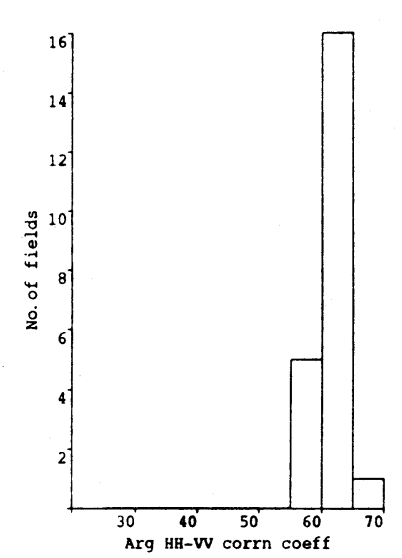

(a)

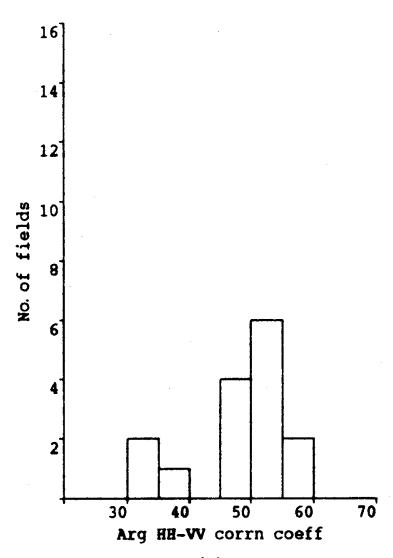

(c)

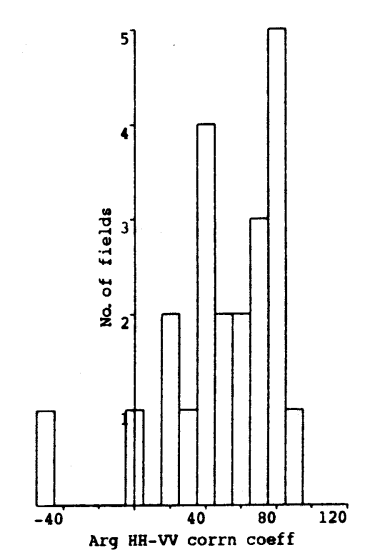

(b)

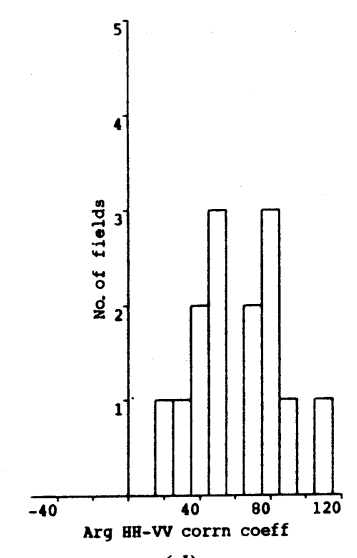

(d)
Figure 9. Histograms of the measured phase of the $H H-V V$ complex correlation coefficient, as a function of crop type and frequency. Figures $9(a)$ and $(c)$ show $C$ band
results for sugar beet and wheat, respectively, figures $9(b)$ and $(d)$ show the corresponding results for $P$ band.

overlap between the sugar beet and wheat measurements, but their phase behaviour is clearly different.

At $P$ band the story is quite different. Sugar beet values lie mainly in the range $20-80^{\circ}$, but two fields lie outside this group, one with a value as low as $-55^{\circ}$. Whea values are spread fairly uniformly across the range $20-180^{\circ}$. When the sample size is taken into account, these histograms give no evidence that sugar beet and wheat ive rise to different phase difference responses at $P$ band. It was also observed theat give $P$ nise to difernt band backscattered power provided no discriminating power between these two crop from the soil. as seems likely at this long wavelength.
8. Conclusions

Observations from the MAESTRO campaign over agricultural regions support the hypothesis that at $C$ band the complex scattering amplitudes of such targets can be modelled by a complex multi-variate mean-zero Gaussian process. The information carried by this process is therefore carried by the covariance matrix, and this tion car the by the At $P$ band, should be treas as the pre observations are not consiste covariance matrix in summarising the information carried by the data is therefore
not properly established.

The phase information is corrupted by system effects and noise. An effect of the latter is to widen the phase difference histograms, and hence to increase the uncertainty and reduce the information in phase difference measurements. Since evidence for such effects is apparent only at $C$ band in the phase differences of the cross-polarized channels from targets with low cross-polarized power, it may be of little importance for most purposes. There are also unexplained bands, correspond ing to changing correlation lengths in the $C$ band phase data. It is not known if these have any significant effect on the single-point phase statistics, other than the obvious one of causing varying precision by modifying the number of independent samples available.

The removal of other system effects requires calibration, in which a key step is the assumption that the like and cross-polarized scattering coefficients are uncorrelated for targets displaying azimuthal symmetry. Under this assumption, only the copolarized phase differences are then of interest. In this case, the shape of the phase difference phase differes difere histogram is unaffected by calibration, and in fact can be derived from amplitude-only data. Correct absolute values of phase difference requires two angles to be supplied, one of which can be measured from the data, while the other requires phase differences to be known for targets in the image. There seems no acceptable priori basis on which to do this reliably for this dataset, so that the calibratio has been avoided. This does not affect any of the conclusions.

The cyclic nature of phase and properties of the theoretical phase difference distribution imply that the mean and standard deviation are not the appropriate parameters to describe phase statistics. The mode of the distribution is the mos meful measure of a representative value, and measures of spread should be centred bout the mode. When the Gaussian model is valid, such as at $C$ band, the phase of te complex correlation coefficient coincides with this mode. Its amplitude gives a measure of the width of the distribution, and hence how sharply defined the phase fifierence is. At $P$ band, where the Gaussian model is not appropriate, the complex correlation coefficient is not a reliable guide either to the mode or width of the phase difference distribution. These issues will be particularly important at longer waveengths in forested areas, where the prevalent scattering mechanisms can lead to phase differences near $180^{\circ}$ and histograms which are not sharply peaked. Under conditions, the mean and standard deviation are at their most misleading in their description of phase behaviour.

consequence of the Gaussian model at $P$ band needs explanation. It may be further possibility theing only a few wavelengths across a resolution cell, with the number of scatterin the scattering may be dominated by a comparatively small developed. This is currently under investigation. Number fluctuations within the 
resolution cells are not likely to be sufficient to explain the failure of the Gaussian model. A study by Yueh et al. (1990) indicates that (under certain assumptions) these lead to $\mathrm{K}$ distributions, for which the phase difference distributions would be the same as for the Gaussian model.

There is clear evidence for the discriminating power of phase amongst agricultural targets at $C$ band. However, only two crop types have been considered, at a single date, and a far more substantial dataset needs to be examined before the utility of phase as a crop classifier can be established. $P$ band phase difference exhibit no ability to differentiate between the crop types examined here. This lack of discrimination at $P$ band would be expected if the return is dominated by the soil, as expected at this long wavelength.

\section{Acknowledgments}

The authors would like to thank the Joint Research Centre, Ispra, and the European Space Agency for the supply of data used in this paper; they would also like to thank Huntings Technical Services for the supply of ground data

\section{References}

Davenport, W. B., JR., and Root, W. L., 1958, An introduction to the theory of random signals and noise (New York: McGraw Hill Book Co.).

signals and noise (New York: McGraw Hill Book Co.).
Dutra, L. V., QuEGAN images. Proceedings of IGARSS 91, Espoo, Finland, I.E.E.E. Catalog Number 91CH2971-0, Library of Congress Number 90-86187, pp. 345-348.

KLein, J. D., and Freman, A., 1991, Quadpolarization SAR calibration using targcl reciprocity. Journal of Electromagnetic Waves and Applications, 5, 735-751.

Kuga, Y., WhitT, M. W., MCDonald, K. C., and UlabY, F. T., 1990, Scattering models for distributed targets. In Radar polarimetry for geoscience applications, edited by $\mathrm{F} . \mathrm{T}$ Ulaby and C. Elachi (Norwood, MA: Artech House Inc.), pp. 111-190.

LeE, J., Grunes, M. R., and MANGo, S. A., 1991, Speckle reduction in multipolarisation multifrequency SAR imagery. I.E.E.E. Transactions on Geoscience and Remote Sensing,

Nghiem, S. V., YUeh, S. H., Kwok, R., and LI, F. K., 1992, Symmetry properties in polarimetric remote sensing. Radio Science, 27, 693-711.

Press, W. H., Flannery, B. P., Teukolsky, S. A., and VetTerling, W. T., 1986, Numerical Recipes (Cambridge, New York, Melbourne: Cambridge University Press)

QUEGAN, S., 1992, Cross-talk calibration of the MAESTRO data from the Reedham and Feltwell test sites. MAESTRO/AGRISCATT: Radar techniques for forestry and agriculNetherlands, 6-7. Proceedings of the Final Workshop held in ESTEC, Noordwitk. The pp. 35-40. 6-7 March, 1992,

SARABANDI, K., 1992, Derivation of phase statistics from the Mueller matrix. Radio Science, 27, 553-560.

VAN ZYL, J. J., 1990, Calibration of polarimetric radar images using only image parameter and trihedral corner reflector responses. I.EE.E. Transactions on Geoscience und Remote Sensing, 28, 337-348.

Yueh, S. H., Kong, J. A., Jao, J. K., Shin, R. T., Zebker, H. A., Le Toan, T., and OMII. II. 1990, K-distribution and polarimetric terrain radar clutter. In Polarimetric remore sensing, edited by J. A. Kong (Amsterdam: Elsevier Science Publishing (o.) pp. 237-275.

ZEBKER, H. A., and Lou, Y., 1990, Phase calibration of imaging radar polarimeter Stoke matrices. I.E.E.E. Transactions on Geoscience and Remote Sensing, 28, 246-252. 